\title{
Extended screening for major mitochondrial DNA point mutations in patients with hereditary hearing loss
}

\author{
Tomofumi Kato ${ }^{1,2}$, Yutaka Nishigaki ${ }^{2}$, Yoshihiro Noguchi ${ }^{3}$, Noriyuki Fuku ${ }^{2}$, Taku Ito ${ }^{3}$, Eri Mikami ${ }^{2}$, Ken Kitamura \\ and Masashi Tanaka ${ }^{2}$ \\ Hearing loss (HL) is the most common sensory disorder in humans. Many patients with mitochondrial diseases have \\ sensorineural HL (SNHL). The HL of these patients manifests as a consequence of either syndromic or nonsyndromic \\ mitochondrial diseases. Furthermore, the phenotypes vary among patients even if they are carrying the same mutation. \\ Therefore, these features make it necessary to analyze every presumed mutation in patients with hereditary HL, but the \\ extensive analysis of various mutations is laborious. We analyzed 373 patients with suspected hereditary $\mathrm{HL}$ by using an \\ extended suspension-array screening system for major mitochondrial DNA (mtDNA) mutations, which can detect 32 other \\ mtDNA mutations in addition to the previously analyzed 29 mutations. In the present study, we detected 2 different mtDNA \\ mutations among these 373 patients; $\mathrm{m} .7444 \mathrm{G}>\mathrm{A}$ in the MT-CO1 gene and m.7472insC in the MT-TS1 gene in 1 patient \\ $(0.3 \%)$ for each. As these two patients had no clinical features other than $\mathrm{HL}$, they had not been suspected of having mtDNA \\ mutations. This extended screening system together with the previous one is useful for the genetic diagnosis and \\ epidemiological study of both syndromic and nonsyndromic $\mathrm{HL}$. \\ Journal of Human Genetics (2012) 57, 772-775; doi:10.1038/jhg.2012.109; published online 13 September 2012
}

Keywords: hereditary hearing loss; mitochondrial DNA; mutation; suspension array

\section{INTRODUCTION}

We can find many patients with hearing loss (HL) among those with mitochondrial DNA (mtDNA) mutations. These patients are classified into two categories; those having only HL (nonsyndromic HL) and those with HL plus other symptoms of mitochondrial disease (syndromic HL). Furthermore, as the severities and phenotypes of mitochondrial diseases vary from patient to patient, we often find mtDNA mutations in unexpected cases. ${ }^{1}$ The fact that there are many cases without any apparent family history makes it more difficult to diagnose mitochondrial diseases. ${ }^{2}$ Sensorineural HL (SNHL) is the most common sensory disorder in humans, having a prevalence of 2.7 per 1000 in children under 5 years of age. ${ }^{3}$ The frequency of patients with HL caused by mtDNA mutations increases with age, because mitochondrial diseases usually become aggravated with age. Therefore, it is necessary to analyze many different suspected mutations in mtDNA, but it is very exhaustive to examine these muations one by one.

Previously, we reported the results of extensive and rapid screening for major 29 major point mutations of mtDNA in patients with hereditary HL by using a suspension array technology. ${ }^{4}$ Our previous survey of 373 patients with suspected HL by use of this screening system revealed the m.1555A $>\mathrm{G}$ mutation in 11 patients, the
m.3243A $>\mathrm{G}$ mutation in 9 patients, and the m.8348A $>\mathrm{G}$, m.11778G $>A$ and m.15498G $>$ A mutations in 1 patient each. In the present extended study, we increased the number of mutations that could be detected from 29 to 61 . We examined the applicability of this extended screening system for genetic diagnosis of hereditary HL by analyzing these same 373 patients with suspected hereditary HL.

\section{MATERIALS AND METHODS}

\section{Patients}

The study population included 373 unrelated Japanese patients with suspected hereditary HL, who visited the outpatient clinic of the Department of Otolaryngology, University Hospital of Medicine, Tokyo Medical and Dental University. The subjects included patients with a family history of HL and those with no apparent cause of HL, even though they did not have any apparent family history of HL. Their detailed demographic and audiometric features are shown in Table 1. The average age of them was 40 years, with a range between 1 and 77 years.

The study protocol complied with the Declaration of Helsinki, and it was also approved by the Committee on the Ethics of Human Research of the Tokyo Metropolitan Institute of Gerontology and the Institutional Review Board (IRB no. 68) of Tokyo Medical and Dental University. This study was carried out only after obtaining the written informed consent of each individual and/or the parents in the case of children.

${ }^{1}$ Department of Otolaryngology, Tokyo Metropolitan Geriatric Hospital and Institute of Gerontology, Tokyo, Japan; ${ }^{2}$ Department of Genomics for Longevity and Health, Tokyo Metropolitan Institute of Gerontology, Tokyo, Japan and ${ }^{3}$ Department of Otolaryngology, Tokyo Medical and Dental University, Tokyo, Japan

Correspondence: Dr M Tanaka, Department of Genomics for Longevity and Health, Tokyo Metropolitan Institute of Gerontology, 35-2 Sakae-cho, Itabashi-ku, Tokyo 173-0015, Japan.

E-mail: mtanaka@tmig.or.jp

Received 27 June 2012; revised 14 August 2012; accepted 15 August 2012; published online 13 September 2012 
Table 1 Demographic features of HL patients

\begin{tabular}{|c|c|}
\hline \multicolumn{2}{|l|}{ Sex } \\
\hline Female (\%) & $229(61.4)$ \\
\hline \multicolumn{2}{|l|}{ Onset age of HL (years) } \\
\hline Newborn or $0(\%)$ & $31(8.3)$ \\
\hline $1 \sim 3(\%)$ & $23(6.2)$ \\
\hline $4 \sim 10(\%)$ & $80(21.4)$ \\
\hline $11 \sim 20(\%)$ & $43(11.5)$ \\
\hline $21 \sim 30(\%)$ & $39(10.5)$ \\
\hline $31 \sim 40$ (\%) & $50(13.4)$ \\
\hline $41 \sim 50(\%)$ & $37(9.9)$ \\
\hline $51 \sim 60(\%)$ & $31(8.3)$ \\
\hline $61 \sim 70(\%)$ & $12(3.2)$ \\
\hline $71 \sim 80(\%)$ & $5(1.3)$ \\
\hline Unknown (\%) & $22(5.9)$ \\
\hline \multicolumn{2}{|l|}{ Mode of inheritance } \\
\hline Autosomal dominant (\%) & $92(24.7)$ \\
\hline Autosomal recessive (\%) & 52 (13.9) \\
\hline Maternal (\%) & $47(12.6)$ \\
\hline X-linked (\%) & 0 \\
\hline Sporadic (\%) & $179(48.0)$ \\
\hline Unknown (\%) & $3(0.8)$ \\
\hline \multicolumn{2}{|l|}{ Type of audiogram } \\
\hline High-frequency steeply sloping (\%) & $80(21.4)$ \\
\hline High-frequency gently sloping (\%) & $104(27.9)$ \\
\hline Flat (\%) & 39 (10.5) \\
\hline U-shaped (Cookiebite) (\%) & $39(10.5)$ \\
\hline Reverse U-shaped (\%) & $4(1.1)$ \\
\hline Low frequency $(\%)$ & $39(10.5)$ \\
\hline Deafness $(\%)$ & $21(5.6)$ \\
\hline Others $(\%)$ & $43(11.5)$ \\
\hline Unknown (\%) & $4(1.1)$ \\
\hline Total (\%) & $373(100)$ \\
\hline
\end{tabular}

Abbreviation: $\mathrm{HL}$, hearing loss.

Extended screening of mtDNA pathological mutation by use of suspension-array technology

DNA samples were purified from the blood by using a standard procedure. The mtDNA from each patient was analyzed with the previously described extended suspension array-based screening system. ${ }^{5}$ The targets of the present analysis were $32 \mathrm{mtDNA}$ mutations in 15 genes: 1 in each of MT-TQ (tRNA $\left.{ }^{\text {Gln }}\right), M T-T W\left(t R N A^{T r p}\right), M T-T C\left(t R N A^{C y s}\right), M T-T H\left(t R N A^{H i s}\right)$, MT-TL2 (tRNA $\left.{ }^{\operatorname{Leu}(C U N)}\right), M T-N D 6$ and MT-CYB genes; 2 in each of MT-TN $\left(t R N A^{A s n}\right), M T-A T P 6, M T-T G\left(t R N A^{G l y}\right)$ and MT-TE (tRNA $\left.{ }^{G l u}\right)$ genes; 3 in the $M T-N D 3$ gene; 4 in the MT-CO1 gene; and 5 in each of the MT-TI $\left(t R N A^{\text {Ile }}\right)$ and MT-TS1 $\left(t R N A^{\operatorname{Ser}(U C N)}\right)$ genes as shown in Table 2. The mtDNA mutations reported in our previous study were within the DNA fragments amplified by multiplex PCR for mtDNA haplotyping, which was mainly designed for anthropological purposes. For the present study, we newly designed a second multiplex PCR system to analyze the 32 additional mtDNA mutations (including m.7445, which is known to cause of HL).

\section{Comparison of results between suspension array and direct DNA} sequencing

DNA sequencing was carried out by using an Applied Biosystems $3130 \times 1$ Genetic Analyzer (Applied Biosystems, Foster City, CA, USA), a BigDye Terminator v3.1 Cycle Sequencing Kit (Applied Biosystems) and Sequencher version 4.2.2 (Gene Codes, Ann Arbor, MI, USA) to compare the sequences with the revised Cambridge reference sequence, ${ }^{6,7}$ while following the standard procedure. ${ }^{8,9}$
Table 2 List of 32 mutations examined by use of the extended suspension array-based system for the detection of mtDNA mutation detection system

\begin{tabular}{|c|c|c|c|c|}
\hline $\begin{array}{l}\text { Nucleotide } \\
\text { position (m) }\end{array}$ & $\begin{array}{c}\text { Nucleotide } \\
\text { change }\end{array}$ & $\begin{array}{l}\text { Amino } \\
\text { acid } \\
\text { change }\end{array}$ & Locus & Clinical phenotype \\
\hline 4269 & $A>G$ & & MT-TI & Encephalopathy/FICP \\
\hline 4295 & $A>G$ & & MT-TI & MHCM \\
\hline 4298 & $\mathrm{G}>\mathrm{A}$ & & MT-TI & CPEO/MS \\
\hline $4300^{a}$ & $A>G$ & & MT-TI & MICM \\
\hline 4320 & $\mathrm{C}>\mathrm{T}$ & & MT-TI & MHCM \\
\hline 4332 & $G>A$ & & MT-TQ & MELAS/encephalopathy \\
\hline 5537 & $A>$ ins $T$ & & MT-TW & MILS \\
\hline 5698 & $\mathrm{G}>\mathrm{A}$ & & MT-TN & CPEO/MM \\
\hline 5703 & $G>A$ & & MT-TN & CPEO/MM \\
\hline 5814 & $\mathrm{~T}>\mathrm{C}$ & & MT-TC & Encephalopathy \\
\hline $7443^{b}$ & $A>G$ & Ter-G & MT-CO1 & DEAF \\
\hline $7444^{b}$ & $G>A$ & Ter-K & MT-CO1 & LHON/SNHL/DEAF \\
\hline $7445^{b}$ & $A>C$ & Ter-S & MT-CO1 & DEAF \\
\hline $7445^{a}$ & $A>G$ & Ter-Ter & MT-CO1 & SNHL \\
\hline $7472^{b}$ & $C>$ ins $C$ & & MT-TS1 & PEM/AMDF \\
\hline $7497^{a}$ & $G>A$ & & MT-TS1 & MM/exercise intolerance \\
\hline 7510 & $\mathrm{~T}>\mathrm{C}$ & & MT-TS1 & SNHL \\
\hline $7511^{a}$ & $\mathrm{~T}>\mathrm{C}$ & & MT-TS1 & SNHL \\
\hline $7512^{a}$ & $\mathrm{~T}>\mathrm{C}$ & & MT-TS1 & PEM/MERRF + MELAS \\
\hline 8993 & $\mathrm{~T}>\mathrm{C}$ & $L>P$ & MT-ATP6 & NARP/MILS \\
\hline 8993 & $\mathrm{~T}>\mathrm{G}$ & $L>R$ & MT-ATP6 & NARP/MILS \\
\hline 9997 & $\mathrm{~T}>\mathrm{C}$ & & MT-TG & MHCM \\
\hline 10010 & $\mathrm{~T}>\mathrm{C}$ & & MT-TG & PEM \\
\hline $10158^{a}$ & $\mathrm{~T}>\mathrm{C}$ & $S>P$ & MT-ND3 & MILS \\
\hline 10191 & $\mathrm{~T}>\mathrm{C}$ & S-P & MT-ND3 & ESOC/Leigh-like disease/MILS \\
\hline $10197^{a}$ & $G>A$ & $A-T$ & MT-ND3 & MILS/dystonia/stroke \\
\hline 12147 & $G>A$ & & MT-TH & MERRF + MELAS/cerebral edema \\
\hline 12297 & $\mathrm{~T}>\mathrm{C}$ & & MT-TL2 & Dilated cardiomyopathy \\
\hline $14568^{b}$ & $\mathrm{C}>\mathrm{T}$ & G-S & MT-ND6 & LHON \\
\hline $14709^{a}$ & $\mathrm{~T}>\mathrm{C}$ & & MT-TE & MM + DMDF/encephalomyopathy \\
\hline 14710 & $G>A$ & & MT-TE & Encephalomyopathy + retinopathy \\
\hline 15243 & $G>A$ & $G>E$ & MT-CYB & MHCM \\
\hline
\end{tabular}

Abbreviations: AMDF, ataxia, myoclonus and deafness; ATP6, ATP synthase $F_{0}$ subunit 6 ; $\mathrm{CO1}$, cytochrome $c$ oxidase subunit I; CPEO, chronic progressive external ophthalmoplegia; CYB, cytochrome $b$; DEAF, maternally inherited deafness or aminoglycoside-induced deafness; DMDF, diabetes mellitus + deafness; ESOC, epilepsy, strokes, optic atrophy and cognitive decline; FICP, fatal infantile cardiomyopathy, plus a MELAS-associated cardiomyopathy; LHON, Leber hereditary optic neuropathy; MELAS, mitochondrial myopathy, encephalopathy, lactic acidosis and stroke-like episodes; MERRF, myoclonic epilepsy and ragged-red fibers; MHCM, maternally inherited hypertrophic cardiomyopathy; MICM maternally inherited MHCM, maternally inherited hypertrophic cardiomyopathy; MICM maternally inherited
cardiomyopathy; MILS, maternally inherited Leigh syndrome; MR, mental retardation; MS, multiple sclerosis; mtDNA, mitochondrial DNA; NARP, neurogenic muscle weakness, ataxia and retinitis pigmentosa; ND, NADH dehydrogenase subunit; SNHL, sensorineural hearing loss; PEM, progressive encephalomyopathy.

Abbreviations and information about mutations are annotated in the MITOMAP database. a Mutation reported as both homoplasmic and heteroplasmic.

bMutation reported as homoplasmic.

\section{RESULTS AND DISCUSSION}

In the present extended study, 2 of the 32 mtDNA mutations, m.7444G $>$ A and m.7472insC, were detected by the screening system, each in 1 patient out of the 373 patients with SNHL. The median fluorescent intensities for the m.7444G $>\mathrm{A}$ mutation and the 7472ins C mutation are displayed in scatter diagrams (Figure 1). When the median fluorescent intensity values for the wild-type signals were below the cut-off values, we regarded the mutations as homoplasmic. The m.7444G $>$ A mutation was homoplasmic and the 7472insC mutation was heteroplasmic. On the basis of the 

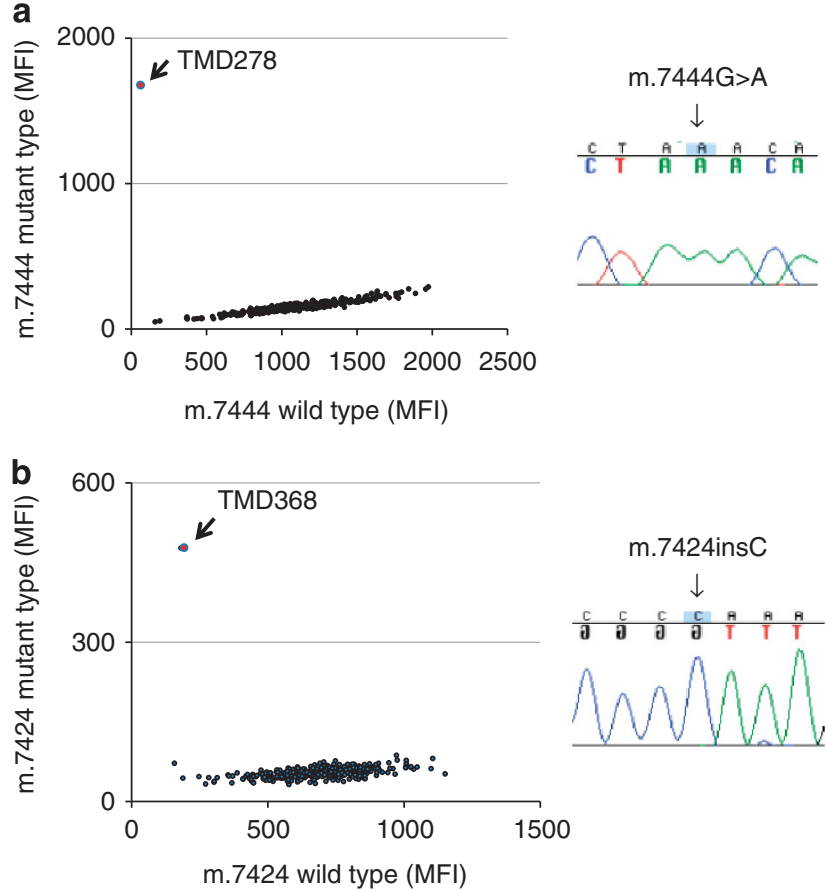

Figure 1 Scatter diagrams with mutant median fluorescent intensity values on the $y$ axis and wild-type ones on the $x$ axis and electropherograms of DNA sequences for the m.7444G $>$ A homoplasmic mutation (a) and the m.7472insC heteroplasmic mutation (b). All 373 DNA samples were analyzed by the m.7444G $>A$ and m.7472insC mutation detection systems, using universal 96-well plates. Later on, each result was merged into the two separate scatter diagrams. Red circles indicate median fluorescent intensity values for mutation-positive DNAs.

Table 3 Mitochondrial DNA mutations detected in 373 patients with hereditary HL screened by the previous and present detection system

\begin{tabular}{lcc}
\hline$m$ mtDNA mutation & Number & Frequency (\%) \\
\hline $\begin{array}{l}\text { Previous study (Kato, 2010) } \\
\text { m.1555A }>\text { G }\end{array}$ & 11 & 2.9 \\
m.3243A > G & 9 & 2.4 \\
m.8348A > G & 1 & 0.3 \\
m.11778G > A & 1 & 0.3 \\
m.15498G > A & 1 & 0.3 \\
Present study & & \\
m.7444G $>$ A & 1 & 0.3 \\
m.7472insC & 1 & 0.3 \\
Undetected & 348 & \\
Total & 373 & 93.3 \\
\hline
\end{tabular}

Abbreviation: $\mathrm{HL}$, hearing loss.

chromatogram, the mutation load was estimated as $59 \%$. None of the other 30 mutations were detected in these patients with SNHL. We summarized the mtDNA mutations detected by the previous and the present analytical systems in Table 3 .

SNHL is one of the most common disorder in patients with mitochondrial diseases, ${ }^{10}$ which is represented by the mutations of the homoplasmic m.1555A $>\mathrm{G}$ and the heteroplasmic m.3243A $>$ G. ${ }^{11-16}$ We previously reported that not only these mutations but also other a

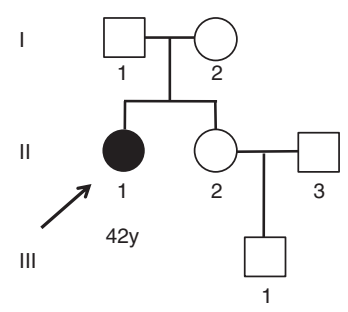

b
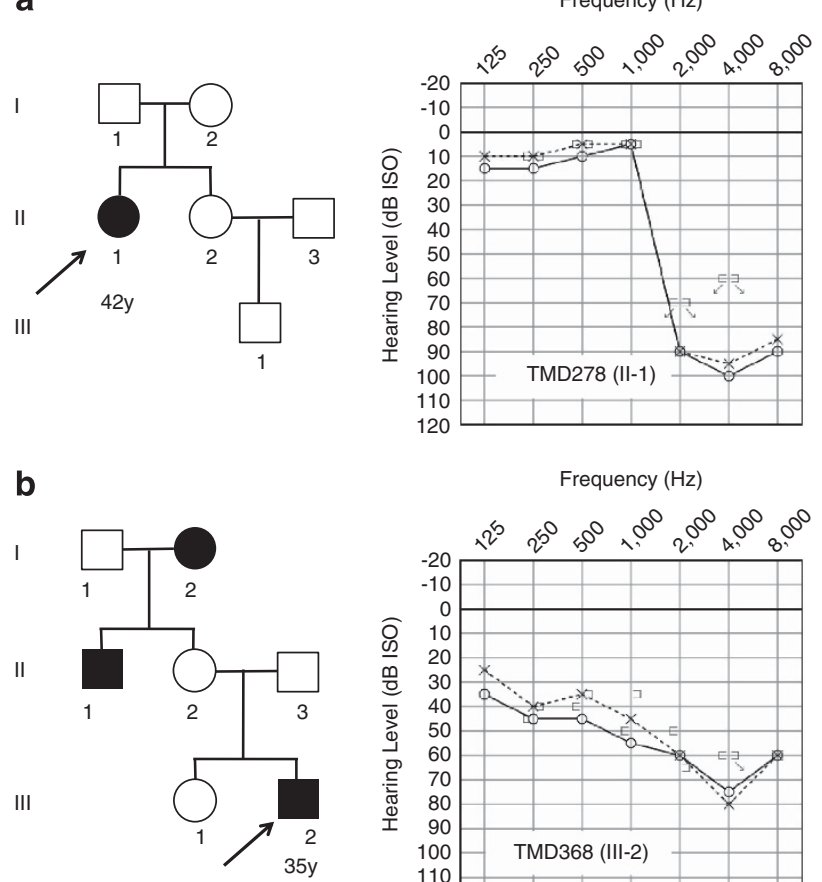

Frequency $(\mathrm{Hz})$

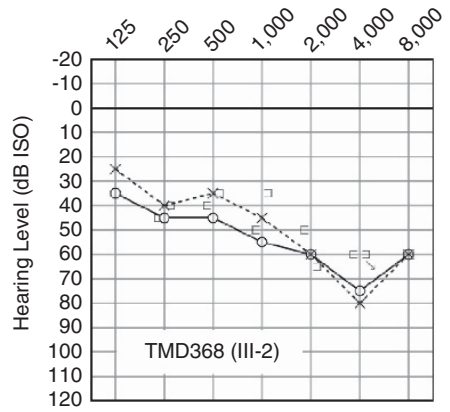

Figure 2 Pedigrees of the families and audiograms of patient TMD278 (a) and patient TMD368 (b). Clinical features are depicted: black-filled circles or squares as individuals with deafness. Arrows indicate probands. Symbols on pure tone audiograms: $\mathrm{dB}$, decibels; ISO, international standards organization. ], left-ear bone conduction; [, right-ear bone conduction; O, right-ear air conduction; $\mathrm{X}$, left-ear air conduction.

mutations could be detected in the patients with either nonsyndromic or syndromic hereditary HL. The m.7444G $>$ A mutation was earlier reported as a cause of aminoglycoside-induced and nonsyndromic HL. ${ }^{17}$ However, patient TMD278, carrying this mutation, had no history of aminoglycoside injection in her detailed clinical history. On the other hand, we should mention that this mutation characterizes haplogroup V7 and H40b, and there is no direct evidence that these haplogroups tend to have HL. ${ }^{18}$ Furthermore, it was also reported that the m.7444G $>$ A mutation is a secondary mutation found in patients with Leber's hereditary optic neuropathy (LHON) and that this mutation has an additional role in the pathogenesis of LHON. ${ }^{19,20}$ Primary mutations, m.11778G $>$ A, m.3460G $>$ A and m.14484T $>\mathrm{C}$ can cause LHON. However, these mutations had already been examined in our previous study and the patient TMD278 was negative for them. With regard to her clinical data, she had high-tone SNHL as shown in Figure 2a, although she was 42 years of age. The onset of her HL occurred during her childhood, after which the HL became progressive. She had started wearing hearing aids 3 years before visiting our clinic. Her clinical feature seemed sporadic because she had neither other clinical disorders such as LHON nor a family history of HL.

The m.7472insC was reported as a pathogenic heteroplasmic mutation within the coding region of tRNA ${ }^{\mathrm{Ser}(\mathrm{UCN})}$. This mutation was previously reported to be associated with progressive myoclonus epilepsy or syndromic disorders including HL, ataxia and myoclonus in previous reports. ${ }^{21}$ The phenotypes of this mutation, however, vary even among individuals within the same family. ${ }^{22}$ The audiogram of TMD368 showed moderate SNHL (Figure 2b), although this patient 
was still just 35 years old. He had no other clinical disorders such as myoclonus or family history of $\mathrm{HL}$ or neurological disorders. It should also be noted that recently the m.7472insC mutation was identified in gastric cancer tissues. ${ }^{23}$ This report suggested that somatic mtDNA mutations may have an important role in the progression of gastric cancer.

In conclusion, the present extended screening system by use of a suspension array for major mtDNA mutations was demonstrated to be powerful, because we could detect both major causative and unexpected mtDNA mutations. The present system is helpful for both the diagnosis and epidemiological studies. Detecting mtDNA mutations in the early stage of HL could be meaningful both to select the optimal therapeutic strategies for the patients and to provide appropriate genetic counseling.

\section{ACKNOWLEDGEMENTS}

We thank Y Abe for helpful discussions and excellent technical support. This work was supported in part by grants from the programs grants-in-aid for young scientists (B)-(no. 22791577 to TK), grants-in-aid for scientific research (B)-(no. 21390459 to KK), grants-in-aid for scientific research (C) (no. 18590317 to Y Nishigaki and no. 21590411 to HH) and grants-in-aid for scientific research (A-22240072, B-21390459 and C-21590411 to MT) from the Ministry of Education Culture, Sports, Science and Technology; by a grant-in-aid for scientific research from the Ministry of Health, Labor and Welfare of Japan (H23-kankaku-005 to KK); by grants-in-aid for the Research on Intractable Diseases (Mitochondrial Disease H23-016 and H23-119) from the Ministry of Health, Labor, and Welfare (to MT); and by grants for scientific research from the Takeda Science Foundation (to MT).

1 Schapira, A. H. Mitochondrial disease. Lancet 368, 70-82 (2006).

2 DiMauro, S. \& Schon, E. A. Mitochondrial respiratory-chain diseases. N. Engl. J. Med. 348, 2656-2668 (2003).

3 Morton, C. C. \& Nance, W. E. Newborn hearing screening-a silent revolution. N. Engl. J. Med. 354, 2151-2164 (2006).

4 Kato, T., Nishigaki, Y., Noguchi, Y., Ueno, H., Hosoya, H., Ito, T. et al. Extensive and rapid screening for major mitochondrial DNA point mutations in patients with hereditary hearing loss. J. Hum. Genet. 55, 147-154 (2010).

5 Nishigaki, Y., Ueno, H., Coku, J., Koga, Y., Fujii, T., Sahashi, K. et al. Extensive screening system using suspension array technology to detect mitochondrial DNA point mutations. Mitochondrion 10, 300-308 (2010).
6 Anderson, S., Bankier, A. T., Barrell, B. G., de Bruijn, M. H., Coulson, A. R., Drouin, J. et al. Sequence and organization of the human mitochondrial genome. Nature 290, 457-465 (1981)

7 Andrews, R. M., Kubacka, I., Chinnery, P. F., Lightowlers, R. N., Turnbull, D. M. \& Howell, N. Reanalysis and revision of the Cambridge reference sequence for human mitochondrial DNA. Nat. Genet. 23, 147 (1999).

8 Nishigaki, Y., Marti, R., Copeland, W. C. \& Hirano, M. Site-specific somatic mitochondrial DNA point mutations in patients with thymidine phosphorylase deficiency. J. Clin. Invest. 111, 1913-1921 (2003).

9 Ueno, H., Nishigaki, Y., Kong, Q. P., Fuku, N., Kojima, S., Iwata, N. et al. Analysis of mitochondrial DNA variants in Japanese patients with schizophrenia. Mitochondrion 9, 385-393 (2009).

10 Xing, G., Chen, Z. \& Cao, X. Mitochondrial rRNA and tRNA and hearing function. Cell Res. 17, 227-239 (2007).

11 Prezant, T. R., Agapian, J. V., Bohlman, M. C., Bu, X., Oztas, S., Quu, W. Q. et al. Mitochondrial ribosomal RNA mutation associated with both antibiotic-induced and non-syndromic deafness. Nat. Genet. 4, 289-294 (1993).

12 Noguchi, Y., Yashima, T., Ito, T., Sumi, T., Tsuzuku, T. \& Kitamura, K. Audiovestibular findings in patients with mitochondrial A1555G mutation. Laryngoscope 114, 344-348 (2004).

13 Goto, Y., Nonaka, I. \& Horai, S. A mutation in the tRNA(Leu)(UUR) gene associated with the MELAS subgroup of mitochondrial encephalomyopathies. Nature 348, 651-653 (1990).

14 Tamagawa, Y., Kitamura, K., Hagiwara, H., Ishida, T., Nishizawa, M., Saito, T. et al. Audiologic findings in patients with a point mutation at nucleotide 3,243 of mitochondrial DNA. Ann. Otol. Rhinol. Laryngol. 106, 338-342 (1997).

15 Oshima, T., Ueda, N., Ikeda, K., Abe, K. \& Takasaka, T. Hearing loss with a mitochondrial gene mutation is highly prevalent in Japan. Laryngoscope 109, 334-338 (1999).

16 Vandebona, H., Mitchell, P., Manwaring, N., Griffiths, K., Gopinath, B., Wang, J. J. et al. Prevalence of mitochondrial $1555 \mathrm{~A}->\mathrm{G}$ mutation in adults of European descent. N. Engl. J. Med. 360, 642-644 (2009).

17 Zhu, Y., Qian, Y., Tang, X., Wang, J., Yang, L., Liao, Z. et al. Aminoglycoside-induced and non-syndromic hearing loss is associated with the G7444A mutation in the mitochondrial COI/tRNASer(UCN) genes in two Chinese families. Biochem. Biophys. Res. Commun. 342, 843-850 (2006).

18 Yao, Y. G., Salas, A., Bravi, C. M. \& Bandelt, H. J. A reappraisal of complete mtDNA variation in East Asian families with hearing impairment. Hum. Genet. 119, 505-515 (2006).

19 Brown, M. D., Voljavec, A. S., Lott, M. T., MacDonald, I. \& Wallace, D. C. Leber's hereditary optic neuropathy: a model for mitochondrial neurodegenerative diseases. FASEB J. 6, 2791-2799 (1992).

20 Matsumoto, M., Hayasaka, S., Kadoi, C., Hotta, Y., Fujiki, K., Fujimaki, T. et al. Secondary mutations of mitochondrial DNA in Japanese patients with Leber's hereditary optic neuropathy. Ophthalmic Genet. 20, 153-160 (1999).

21 Tiranti, V., Chariot, P., Carella, F., Toscano, A., Soliveri, P., Girlanda, P. et al. Maternally inherited hearing loss, ataxia and myoclonus associated with a novel point mutation in mitochondrial tRNASer(UCN) gene. Hum. Mol. Genet. 4, 1421-1427 (1995).

22 Jaksch, M., Klopstock, T., Kurlemann, G., Dorner, M., Hofmann, S., Kleinle, S. et al. Progressive myoclonus epilepsy and mitochondrial myopathy associated with mutations in the tRNA(Ser(UCN)) gene. Ann. Neurol. 44, 635-640 (1998).

23 Hung, W. Y., Wu, C. W., Yin, P. H., Chang, C. J., Li, A. F., Chi, C. W. et al. Somatic mutations in mitochondrial genome and their potential roles in the progression of human gastric cancer. Biochim. Biophys. Acta. 1800, 264-270 (2010). 